\title{
Predicted Useful Lifetime of Aerospace Electronics Experiencing Ionizing Radiation: Application of BAZ Model
}

\author{
A Ponomarev ${ }^{1}$ and E Suhir ${ }^{1,2 *}$ \\ ${ }^{1}$ ERS Co., Los Altos, USA \\ ${ }^{2}$ Portland State University, Portland, USA
}

\begin{abstract}
The objective of the analysis is to demonstrate how the Boltzmann-Arrhenius-Zhurkov (BAZ) model, originally suggested by Zhurkov in the kinetic concept of the strength of solids as a generalization of the Arrhenius theory of chemical reactions, can be effectively employed for the prediction of the lifetime of electronic materials experiencing ionizing radiation. The "loading term" $\gamma \sigma$ in the original BAZ model, where $\sigma$ is the tensile mechanical stress and $\gamma$ is the sensitivity factor, is replaced with the term $\gamma_{R} D$, where $D$ is the radiation level and $\gamma_{R}$ is the sensitivity factor. Leakage current measured during the failure-oriented-accelerated-testing (FOAT) is considered in our analysis as a suitable indication/criterion of the level of the induced damage. FOAT terminates, when the agreed upon critical value of the leakage current is reached.
\end{abstract}

The space environment poses an increased risk of failure for electronic and photonic devices (see, e.g., [1-5]). This analysis is an attempt to quantify, on the probabilistic basis, the outcome of a FOAT [6] and to predict the never-zero probability of failure of the material or the device of interest in the actual operation conditions. It is suggested that the flexible and physically meaningful BAZ equation $[7,8]$ that was used previously for a number of applications in microelectronics and photonics reliability problems (see, e.g., $[9,10]$ ) is used as the model of choice in the probabilistic design for reliability (PDfR) [11] and FOAT efforts. The multi-parametric BAZ model extends the original Zhurkov model for the situations, when the stressor is not tensile mechanical stress, but any other stimulus that contributes to the degradation of the material or the device (such as, e.g., elevated voltage, electrical current, humidity, temperature, vibrations, light output, etc.) and, since the superposition principle does not work in the reliability engineering, - for the situations, when multiple stressors are applied. Another modification of the Zhurkov model is replacement of the time constant $\tau_{0}$ (see eq. (1) below) with an expression that considers the role of time and the parameter that characterizes in a particular problem the degree of degradation. In the situation in question it is the leakage current [12].

BAZ equation for the mean-time-to-failure (MTTF) can be written for the case, when the external loading is ionizing radiation, as follows:

$$
\tau=\tau_{0} \exp \left(\frac{U_{0}-\gamma_{R} D}{k T}\right)
$$

In this equation $\tau_{0}$ is the time constant, $U_{o^{\prime}} e V$, is the basic activation energy that characterizes the propensity of the material or the device to the action of the ionizing radiation, $T^{0}{ }^{0} \mathrm{~K}$ is the absolute temperature, $k=8.61733 \times 10^{-5} \mathrm{eV} / \mathrm{K}$ is Boltzmann's constant, $D, \mathrm{~Gy}=\mathrm{J} / \mathrm{kg}$, is radiation and $\gamma_{R}$ is the sensitivity factor for the case of radiation "stressor". If the exponential law of reliability is used, then the following equation for the probability of non-failure in the case of radiation stressor can be obtained:

$$
\begin{aligned}
& P=\exp (-\lambda t)=\exp \left(-\frac{t}{\tau}\right)= \\
& =\left[-\frac{t}{\tau_{0}} \exp \left(\frac{U_{0}-\gamma_{R} D}{k T}\right)\right]
\end{aligned}
$$

In this double exponential probability distribution function it is considered that the failure rate $\lambda$ is inversely proportional to the MTTF $\tau$ The time $\tau_{0}$ in this equation is an empirical parameter that characterizes the situation at failure, and its physical nature could be selected depending on the magnitude of the monitored quantity used as a suitable indication of FOAT failure. If, e.g., the level $I_{*}$ of the leakage

*Corresponding author: E Suhir, ERS Co., 727 Alvina Ct., Los Altos, CA 94024; Portland State University, Portland, OR 97403, USA

Accepted: April 23, 2019

Published online: April 25, 2019

Citation: Ponomarev A, Suhir E (2019) Predicted Useful Lifetime of Aerospace Electronics Experiencing lonizing Radiation: Application of BAZ Model. J Aerosp Eng Mech 3(1):167-169 
current $I$ is accepted as a suitable characteristic of the level of damage, then the time $\tau_{0}$ could be represented as, say, $\tau_{0}=\frac{1}{\gamma_{I} I_{*}}$, where $\gamma_{l}$ is the sensitivity factor for this current. Then the formula (2) can be written as

$$
P=\exp \left[-\gamma_{I} I_{*} t \exp \left(-\frac{U_{0}-\gamma_{R} D}{k T}\right)\right]
$$

There are three unknowns in this formula: two sensitivity factors, $v_{1}$ and $V_{R}$, and the activation energy $U_{0}$. These unknowns can be found using FOAT. Let us show how this could be done.

Testing should be conducted in two steps. At the first step testing should be carried out for the same level of radiation, but for two different temperature levels, $T_{1}$ and $T_{2}$. Then the following two experimental relationships will be obtained:

$$
P_{1,2}=\exp \left[-\gamma_{I} I_{\star} t_{1,2} \exp \left(-\frac{U_{0}-\gamma_{R} D}{k T_{1,2}}\right)\right]
$$

Here $P_{1,2}$ are the measured probabilities of non-failure, $t_{1,2}$ are the corresponding times and $I_{*}$ is the level of the leakage current that is considered as an indication of the radiation related failure. Since the numerator $U_{0}-\nu_{R} D$ in the relationships (4) is kept the same, the factor $\gamma_{1}$ can be found as

$$
\gamma_{I}=\exp \left(\frac{\frac{T_{2}}{T_{1}} \ln n_{2}-\ln n_{1}}{\frac{T_{2}}{T_{1}}-1}\right)
$$

where

$$
n_{1,2}=-\frac{\ln P_{1,2}}{I_{*} t_{1,2}}
$$

After the sensitivity factor $\gamma_{1}$ is determined, the second step of FOAT should be conducted at two different radiation levels to determine the sensitivity factor $\gamma_{R}$. The temperatures at these tests do not have to be the same. Considering that the stress-free activation energy $U_{o}$ should remain the same, the following formula for the sensitivity factor $\gamma_{R}$ can be obtained:

$$
\gamma_{R}=k \frac{T_{1} \ln n_{1}-T_{2} \ln n_{2}+\left(T_{2}-T_{1}\right) \ln \gamma_{I}}{D_{1}-D_{2}},
$$

where the notation (6) is used (the actual numbers are those obtained at this, second, step, and are different, of course, of the numbers obtained at the first step of testing). While the temperatures $T_{1}$ and $T_{2}$ do not have to be the same, the formula (7) could be simplified, if they are kept the same. Then the factor $\gamma_{R}$ can be evaluated as

$$
\gamma_{R}=k T \frac{\ln \left(\frac{n_{1}}{n_{2}}\right)}{D_{1}-D_{2}}
$$

Here $T$ is the testing temperature. In such a situation


sensitivity factors $\gamma_{l}$ and $\gamma_{R}$ are evaluated, the activation energy can be found as

$$
\begin{aligned}
& U_{0=}-k T_{1} \ln \left(\frac{n_{1}}{\gamma_{1}}\right)+\gamma_{R} D_{1}= \\
& =k T_{2} \ln \left(\frac{n_{2}}{\gamma_{1}}\right)+\gamma_{R} D_{2}
\end{aligned}
$$

Let, e.g., the following data have been obtained at the first step of FOAT:

1) After $t_{1}=35 \mathrm{~h}$ of testing at the temperature of $T_{1}=$ $60{ }^{\circ} \mathrm{C}=333^{\circ} \mathrm{K}$ and after the total ionizing dose of $D=1.0 \mathrm{~Gy}=$ $1.0 \mathrm{~J} / \mathrm{kg}$ was obtained, $10 \%$ of the tested devices reached the critical level of the leakage current of $I_{*}=3.5 \mu \mathrm{A}$ and, hence, failed, so that the recorded probability of non-failure is $P_{1}=$ 0.9;

2) After $t_{2}=50 \mathrm{~h}$ of testing at the temperature of $T_{2}=85$ ${ }^{\circ} \mathrm{C}=358^{\circ} \mathrm{K}$ and at the same radiation level, $25 \%$ of the tested samples failed, so that the recorded probability of non-failure is $P_{2}=0.75$.

Then the formulas (6) yield:

$$
\begin{aligned}
& n_{1}=-\frac{\ln P_{1}}{I_{*} t_{1}}=-\frac{\ln 0.9}{3.5 \times 35}=8.6009 \times 10^{-4} \mu A^{-1} h^{-1} ; \\
& n_{2}=-\frac{\ln P_{2}}{I_{*} t_{2}}=-\frac{\ln 0.75}{3.5 \times 50}=16.4390 \times 10^{-4} \mu A^{-1} h^{-1} ; \quad \text { and }
\end{aligned}
$$
the formula (5) results in the following value of the parameter $r_{i}$ :

$$
\begin{aligned}
& \gamma_{I}=\exp \left(\frac{\frac{T_{2}}{T_{1}} \ln n_{2}-\ln n_{1}}{\frac{T_{2}}{T_{1}}-1}\right)= \\
& =\exp \left(\frac{\frac{358}{333} \ln 16.4 \times 10^{-4}-\ln 8.60 \times 10^{-4}}{\frac{358}{333}-1}\right)=
\end{aligned}
$$$$
=9.1836 \mu A^{-1} h^{-1}
$$

At the second step of FOAT one can use, without conducting additional testing, the following information from the first step:

1) After $t_{1}=35 \mathrm{~h}$ of testing at the temperature of $T_{1}=$ $60^{\circ} \mathrm{C}=333^{\circ} \mathrm{K}$ and after the total ionizing dose of $D=1.0 \mathrm{~Gy}=$ $1.0 \mathrm{~J} / \mathrm{kg}$ was obtained, $10 \%$ of the tested devices reached the critical level of the leakage current of $I_{*}=3.5 \mu \mathrm{A}$ and, hence, failed, so that the recorded probability of non-failure is $P_{1}=$ 0.9; but, in addition, conduct FOAT for a different radiation level of, say, $D_{2}=2.0 \mathrm{~Gy}$. Let us assume that the following information has been obtained: but, in addition, FOAT should 
be conducted for a different radiation level.

2) After $t_{2}=10 \mathrm{~h}$ of testing at the same temperature and after the total radiation dose of $D_{2}=2.0 \mathrm{~Gy}, 40 \%$ of the tested samples failed, so that the probability of non-failure is $P_{2}=0.6$.

Then the second formula in (6) yields:

$n_{2}=-\frac{\ln P_{2}}{I_{*} t_{2}}=-\frac{\ln 0.6}{3.5 \times 10}=145.9502 \times 10^{-4} \mu A^{-1} h^{-1}$;

and the equation (8) results in the following $\gamma_{R}$ value:

$$
\begin{aligned}
& \gamma_{R}=k T \frac{\ln \left(\frac{n_{1}}{n_{2}}\right)}{D_{1}-D_{2}}= \\
& =8.61733 \times 10^{-5} \times 333 \frac{\ln \left(\frac{8.6009 \times 10^{-4}}{145.9502 \times 10^{-4}}\right)}{1-2}= \\
& =0.081249 \mathrm{eVG} y^{-1}
\end{aligned}
$$

After the sensitivity factors of the leakage current and the radiation are found, the activation energy can be determined as

$$
\begin{aligned}
& U_{0}=-K T_{1} \ln \left(\frac{n_{1}}{\gamma_{1}}\right)+\gamma_{R} D_{1}=-8.61733 \times 10^{-5} \times 333 \ln \left(\frac{8.6009 \times 10^{-4}}{9.1836}\right)+0.081249= \\
& =0.266178+0.081249=0.3474 \mathrm{ev}
\end{aligned}
$$

The expected time-to-failure (TTF) can be determined from (3) as follows:

$$
t=-\frac{\ln P}{\gamma_{I} I_{*}} \exp \left(\frac{U_{0}-\gamma_{R} D}{k T}\right)
$$

This time depends, of course, on the expected (specified) probability $P$.

If, e.g., the specified probability of non-failure in actual operation conditions is, say, $P=0.999999$, the outside temperature is $T=-150^{\circ} \mathrm{C}=123^{\circ} \mathrm{K}$ and the radiation level is $D$ $=1.0 \mathrm{~Gy}$, then, with the obtained FOAT data we find:

$$
\begin{aligned}
& t=-\frac{\ln 0.999999}{9.1836 \times 3.5} \exp \left(\frac{0.3474-0.081249 \times 1.0}{8.61733 \times 10^{-5} \times 123}\right)= \\
& =25010.57 \mathrm{~h}=2.85 \text { years }
\end{aligned}
$$

Future work should focus on experimental verification of the validity of the suggested model, as well as on the consideration of the combined action of several stressors that make physical sense. This could be done by applying the multi-parametric BAZ [9].

\section{References}

1. Ma TP, Dressendorfer PV (1989) lonizing radiation effects in MOS devices and circuits. John Wiley \& Sons, New York, USA.

2. Schwank J (2002) Total dose effects in MOS devices. Radiation effects-from particles to payloads. Proceedings of the IEEE nuclear and space radiation effects conference, short course.

3. Ponomarev AL, Hussein HF, Kim MH, et al. (2007) NASAdeveloped ProE-based tool for the raytracing of spacecraft geometry to determine radiation doses and particle fluxes in habitable areas of spacecraft and in the human body. Technical Report, NASA/TP-2007-214770.

4. Tapero K, Medchurov O (2012) Some Aspects of Radiation Testing of Optocouplers for Space Application. ISROS Conf., 1-5 Oct., Calgari, Sardinia.

5. Tapero K (2015) Private Communications.

6. Suhir E (2008) Micro- and Opto-Electronic Materials and Structures: Physics, Mechanics, Design, Packaging, Reliability. In: E Suhir, CP Wong, YC Lee, Springer.

7. Zhurkov SN (1965) Kinetic concept of the strength of solids. Int J Fract Mech 1: 311-323.

8. Suhir E, Kang SM (2013) Boltzmann-Arrhenius-Zhurkov (BAZ) model in physics-of-materials problems. Modern Physics Letters B (MPLB) 27: 1330009.

9. Suhir E (2014) Three-step concept in modeling reliability: Boltzmann-Arrhenius-Zhurkov physics-of-failure-based equation sandwiched between two statistical models. Microelectron Reliab 54: 2594-2603.

10. Suhir E (2017) Static fatigue lifetime of optical fibers assessed using Boltzmann- Arrhenius-Zhurkov (BAZ) model. J Mater Sci: Mater El 28: 11689-11694.

11. Suhir E (2010) Probabilistic Design for Reliability. Chip Scale Reviews 14: 24-26.

12. Scarpa A, Pacagnella A, Montera F, et al. (1997) lonizing radiation induced leakage current on ultra-thin gate oxides. IEEE Trans Nucl Sci 44: 1818-1825.

DOI: $10.36959 / 422 / 437$ 